\section{Manejo clínico endodóntico de la variación anatómica conducto en "C" tipo IV en un segundo molar mandibular}

\section{Endodontic clinical management of anatomical variation " $\mathrm{C}$ " shaped root canal type IV in a second mandibular molar}

\begin{abstract}
Resumen
Los conductos en forma de $\mathrm{C}$ son una variación anatómica habitualmente encontrada en los segundos molares mandibulares con raíces fusionadas, presentan una morfología transversal que describe un arco de $180^{\circ}$ o más y un solo orificio acintado. El presente caso clínico describe el tratamiento endodóntico con equipos de última generación de un segundo molar mandibular con conducto en forma de C tipo IV y bifurcación a nivel apical de un paciente de sexo masculino que presenta dolor a la percusión, palpación y que al examen con tomografía computarizada Cone Beam (CBCT) se confirma la variación anatómica de la pieza 47 . El diagnóstico fue: tratamiento previamente iniciado asociado a periodontitis apical sintomática. Se realizó el retratamiento de conductos empleando el preflaring previo a la técnica de preparación biomecánica con el Sistema Reciproc® R25, medicación intraconducto con hidróxido de calcio por 7 días, irrigación pasiva con solución de hipoclorito de sodio y técnica de obturación de onda continúa de calor Beefill 2 en 1 sellando herméticamente el tercio apical. El control fue transcurrido tres años y se evidenció el éxito del tratamiento y su bilateralidad anatómica. Es importante hacer uso de la tecnología durante el diagnóstico y tratamiento para acrecentar la probabilidad de éxito a largo plazo.
\end{abstract}

Palabras clave: Diente molar; Tomografía; Variación anatómica.

\section{Caso Clínico}

Mónica Yizely Llanos Carazas ${ }^{1, a}$, Marisa Jara

Castro $^{1, b}$, José Antonio Alanya Ricalde ${ }^{2, c}$

1 Universidad Nacional Mayor de San Marcos.

Facultad de Odontología. Lima, Perú.

2 Universidad Particular Andina del Cusco.

Facultad de Odontología. Cusco, Perú.

a Especialista en Carielogía y Endodoncia.

${ }^{\mathrm{b}}$ Magister en Estomatología.

${ }^{c}$ Magister en Docencia Universitaria.

\section{Correspondencia:}

Mónica Yizely Llanos Carazas

Correo electrónico: mllanosc1@unmsm.edu.pe

Alameda Ciro Alegría 181- Dpto 506 San Borja,

Lima, Perú.

Coautores:

Marisa Jara Castro

mjarac@unmsm.edu.pe

José Antonio Alanya Ricalde

jalanya@uandina.edu.pe

Conflicto de intereses: Los autores declaran no tener conflictos de interés.

Fuente de financiamiento: Autofinanciado.

Fecha de recepción: 09/07/18

Fecha de aceptación: 31/10/18

\begin{abstract}
The C-shaped root canal system is an anatomical variation usually found in second mandibular molar with fused roots, present a transverse morphology that describes an arc of $180^{\circ}$ or more, with a single taped hole orifice. The present clinical case describes the endodontic treatment with last generation equipments of a mandibular molar with C-shaped type IV and fork at apical level in a male patient that has pain to percussion, palpation and the topographic Cone Beam Tomography (CBCT) examination confirms the anatomical variation of the tooth code 47 . The diagnosis was previously initiated treatment with symptomatic apical periodontitis. The retreatment of the canals using

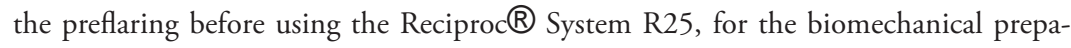
ration technique, calcium hydroxide root canal medication was prescribed for 7 days, sodium hypochlorite as passive irrigation with and continuous heat wave obturation technique beefill 2 in 1 hermetically sealing in apical zone. The control after three years shows the success of technology during the diagnosis and treatment in orden to increase the probability of long term success.
\end{abstract}

Keywords: Anatomic variation; Molar; Tomography.

(C) Los autores. Este artículo es publicado por la revista Odontología Sanmarquina de la Facultad de Odontología, Universidad Nacional Mayor de San Marcos. Este es un artículo de acceso abierto, distribuido bajo los términos de la licencia Creative Commons Atribucion - No Comercia_Compartir Igual 4.0 Internacional. (http://creativecommons.org/licenses/by-nc-sa/4.0/) que permite el uso no comercial, distribución y reproducción en cualquier medio, siempre que la obra original sea debidamente citada. 


\section{Introducción}

El segundo molar mandibular frecuentemente tiene dos raíces, una mesial y otra distal, habitualmente con dos conductos mesiales; mesiobucal (MB) y mesiolingual (ML) y un conducto distal similar a los primeros molares mandibulares, generalmente sus raíces están más juntas, y en muchos casos fusionadas ${ }^{1-3}$.

Las raíces fusionadas que se convierten en una raíz única, cónica o la forma "C-Shaped" tiene una incidencia de aproximadamente $21,8 \%{ }^{3}$.

La configuración en forma de $\mathrm{C}$ del conducto radicular es una de las variaciones anatómicas más importantes ${ }^{4}$, reciben ese nombre debido a la morfología de la sección transversal de su raíz y conducto ${ }^{5}$ y poseen un orificio único en forma de cinta con un arco de $180^{\circ}$ o más ${ }^{6}$.

Kato et al. ${ }^{7}$ mencionan que Keith y Knowles, en Francia, fueron los primeros autores en describir un conducto radicular en forma de $\mathrm{C}$ observado en las secciones transversales de la raíz de un segundo molar mandibular de un individuo Neandertal ${ }^{7}$.

La teoría más aceptada para la formación de la configuración del conducto en forma de $\mathrm{C}$ es la falla de la vaina de la raíz epitelial de Hertwig (HERS) para fusionar la variante anatómica, con la superficie de la raíz bucal $\mathrm{o}$ lingual ${ }^{8}$. Aunque de acuerdo a estudios recientes se atribuye el origen a los genes presentes en los cromosomas 5 o $17^{7}$. La etnicidad juega un papel importante en la formación de conductos en forma de $\mathrm{C}$ y se ve más comúnmente en las poblaciones de China, Corea e India ${ }^{9}$.

A través de los años, se han propuesto distintas clasificaciones de los conductos en forma de C, la primera fue propuesta por Melton et al. ${ }^{10}$ basados en los dos métodos de evaluación, microscopía óptica y estereomicroscopía, propusieron tres niveles arbitrarios. En consecuencia, la morfología de la corona clínica y la apariencia del orificio del conducto pueden no representar la anatomía real del conducto. Las clasificaciones propuestas se compilaron en tres categorías ${ }^{11}$.

- Tipo I: conducto en $C$ completa, define un esquema en forma de $\mathrm{C}$ sin ningún tipo de separación.

- Tipo II: con forma de semicolon o en forma de punto y coma, en los cuales existe dentina separando el conducto distal del vestibular o lingual, la dentina separa un conducto principal en forma de $\mathrm{C}$ de un conducto distinto mesial.

- Tipo III: se refiere a aquellos con dos o más conductos separados o intraconductos.

Posteriormente Fan et al. ${ }^{5-7,12,13}$ modificaron el método de Melton en las siguientes categorías ${ }^{14}$ :

- Clase I (C1): forma de C ininterrumpida, sin separación ni división.

- Clase II (C2): la forma de conducto se parece a un "punto y coma", por interrupción del contor- no de la $C$, pero el ángulo $\alpha$ o $\beta$ no debería ser menor a $60^{\circ}$.

- Clase III (C3): dos o tres conductos separados y los dos ángulos $\alpha$ y $\beta$ son menores de $60^{\circ}$.

- Clase IV(C4): solo un conducto único ovalado en el corte transversal ${ }^{5,7,9,15}$.

- ClaseV (C5): no hay luz que pueda ser observado lo cual suele ser visto cerca del ápice.

Las variaciones de la morfología del conducto radicular, especialmente en dientes multirradiculares, son un desafío constante para el diagnóstico y la terapia endodóntica exitosa. El conocimiento completo de la anatomía del conducto radicular y sus cambios más frecuentes es esencial, ya que la falta de tratamiento en un conducto puede provocar un fracaso en la endodoncia ${ }^{2}$.

El diagnóstico clínico del conducto en forma de C puede reconocerse sobre la base de criterios observables definidos como la anatomía del piso de la cámara pulpar y la persistencia de hemorragia o dolor cuando se localizan los orificios separados del conducto ${ }^{14}$.

Algunos investigadores afirmaron que el diagnóstico radiográfico es difícil; sin embargo, las radiografías preoperatorias nos brindan indicios sobre la morfología de conducto radicular ${ }^{5,16}$.

La tomografía computarizada Cone Beam (CBCT) ha sido ampliamente utilizada en endodoncia en los últimos años debido a su mayor resolución y exploración, así como el tiempo y dosis de radiación reducida ${ }^{2,7}$. Sin embargo, la Asociación Americana de Endodoncia (AAE) y la Academia Americana de Radiología Oral y Maxilofacial (AAOMR), mencionado por Kato et al. ${ }^{7}$ establecen que CBCT sólo debe utilizarse cuando la radiografía dental convencional no permita el diagnóstico adecuado.

\section{Reporte del caso}

Paciente de sexo masculino de 26 años acudió a la clínica de posgrado de la Universidad Nacional Mayor de San Marcos. El paciente refirió dolor intenso previo en la pieza dental 47, por lo que acudió a su odontólogo, quien le realizó el tratamiento de urgencia el día anterior.

A la inspección, se observó material de restauración provisional oclusal y amalgama en vestibular sin evidencia de inflamación de los tejidos blandos.

Se realizaron pruebas complementarias de sensibilidad pulpar: Al test de frío con varilla de hielo (-), al test de calor con gutapercha caliente o método de Grossman $(+)$, a la percusión vertical $(+++)$, palpación $(++)$ y movilidad grado $I$.

Al examen radiográfico, se observó una imagen radiopaca compatible con material de restauración que presentaba aparente compromiso de cámara pulpar y ensanchamiento del espacio para el ligamento periodontal, hueso alveolar conservado, las raíces fusionadas. Se vi- 
sualizó aparentemente un conducto irregular con bifurcación a nivel apical (Figura 1).

El diagnóstico pulpar y periapical fue: tratamiento previamente iniciado - periodontitis apical sintomática. Se estableció como plan de tratamiento el retratamiento no quirúrgico.

Se presumió de la variación anatómica del segundo molar inferior, debido a que presentaba raíces fusionadas y una imagen irregular a nivel del conducto radicular, lo cual fue verificado a través de la CBCT de campo pequeño (Figura 2). Se estableció la variación anatómica del conducto en C clase "IV" de acuerdo a la clasificación de Fan et al. ${ }^{5,7}$ y se utilizó de forma adicional la clasificación propuesta por Vertucci, conducto de tipo $\mathrm{V}$ a nivel apical ${ }^{17}$.

El paciente otorgó su consentimiento informado al plan de tratamiento propuesto, el cual se inició con la eliminación del material restaurador provisional a nivel oclu-

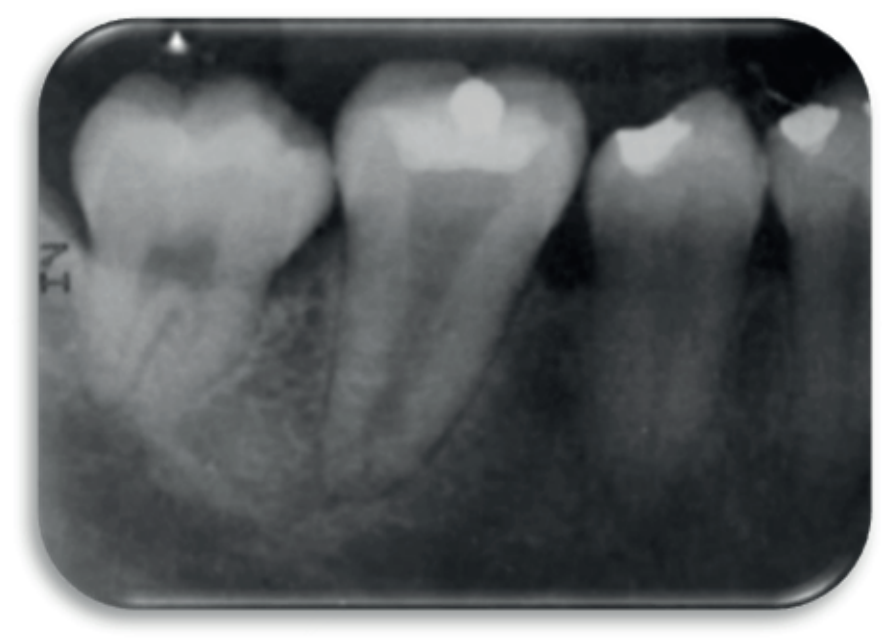

Figura 1. Radiografía inicial pieza 47

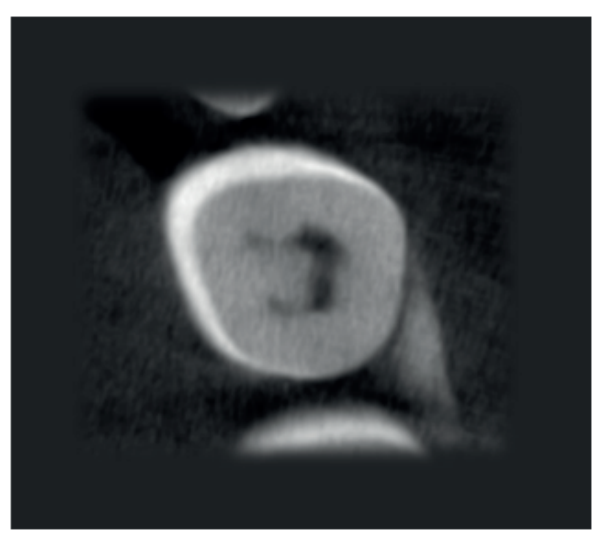

$\mathbf{A}$

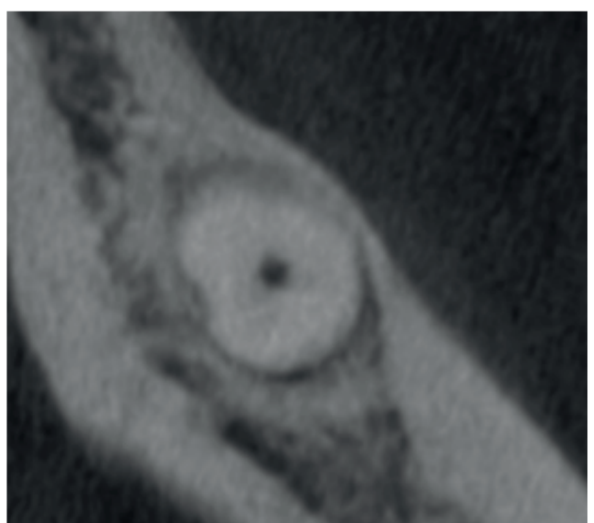

C

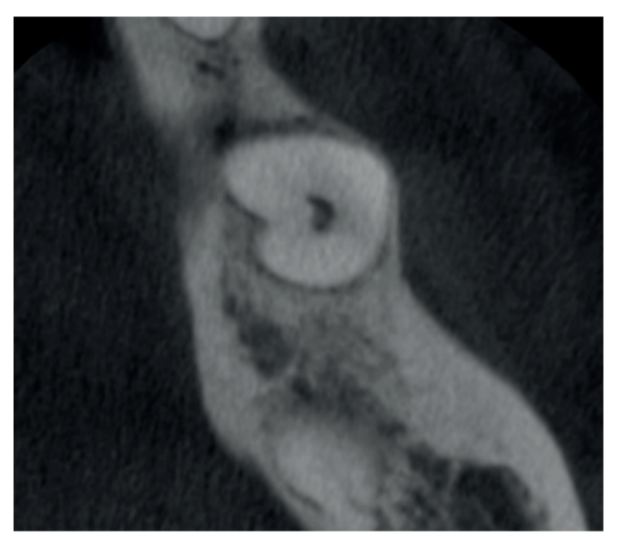

$\mathbf{B}$

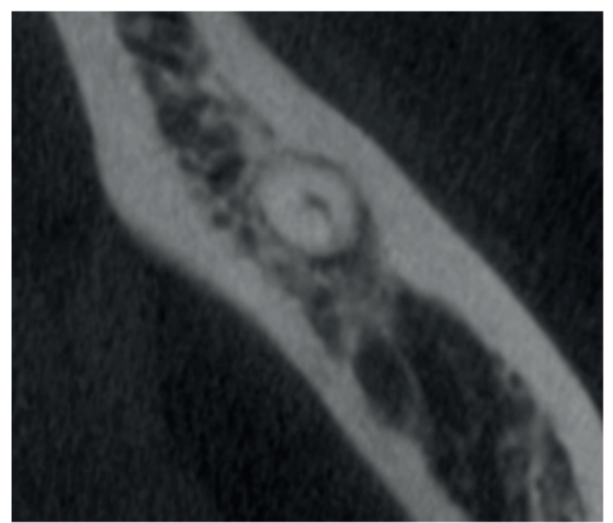

D

Figura 2. Corte Axial CBCT en distintos niveles radiculares. A. Tercio cervical, B. Tercio medio, C. Tercio apical límite de tercio medio y apical y D. Tercio apical 
sal y la apertura cameral bajo aislamiento absoluto del campo operatorio, se visualizó la entrada del conducto de sección transversal que delineaba una $\mathrm{C}$ ininterrumpida con la ayuda de lupas para magnificación $2.5 \mathrm{x}$.

Se exploró los 2/3 radiculares con una lima C-Pilot 10 (VDW-Zipperer, Alemania) a una longitud de $16 \mathrm{~mm}$. El preflaring o ensanchamiento previo fue realizado con la lima Introfile (FlexMaster) accionado con el Motor (VDW Silver Reciproc con un contra ángulo Sirona 6:1) con un número de revoluciones constante entre 280 a $300 \mathrm{rpm}$ de forma pasiva, de acuerdo a las recomendaciones del fabricante. Posteriormente, se realizó la exploración del tercio apical asegurando el Glidepath con una lima C-Pilot 10 (VDW-Zipperer, Alemania) a la longitud de $20 \mathrm{~mm}$, una vez conseguida la guía de deslizamiento se tomó la radiografía de conductometría, cuya longitud fue verificada con el localizador apical RAYPEX® 6 (VDW) estableciendo como longitud de trabajo (LT) $20 \mathrm{~mm}$ (Figura 3). Se finalizó el Glidepath manual con una lima $\mathrm{N}^{\circ} 15$ (Maillefer, Suiza) hasta la longitud de trabajo y la patencia con una lima C-Pilot
10 (VDW-Zipperer, Alemania) a una longitud de 21 $\mathrm{mm}$.

Se utilizó el sistema Reciproc® R25/08 (VDW)® y el motor VDW Silver ${ }^{\circledR}$ Reciproc para la ampliación y conformación del conducto radicular. Se optó por el calibre 25, pues las irregularidades presentes en el sistema de conductos dificultó el acceso de limas de mayor calibre. Posterior a ello, se realizó movimientos de picoteo centrado con progresiones pequeñas repetitivas en dirección apical hasta conseguir la longitud de trabajo alisando las paredes del conducto. Se retiró la lima del conducto, se limpió con una gasa estéril y se verificó la patencia o permeabilidad apical del conducto radicular con una lima de pasaje C-Pilot No 10 (VDW Zipperer, Alemania) a $21 \mathrm{~mm}$, posteriormente se realizaron cinco repeticiones con la lima R25 con abundante solución de hipoclorito de sodio al 2,5\%, se empleó la técnica de irrigación pasiva con una jeringa descartable de $5 \mathrm{ml}$ con aguja Navitip ${ }^{\circledR}$ de color amarillo, la cual se llevó al interior del conducto a $2 \mathrm{~mm}$ de la LT, se secó con puntas de papel absorbente Reciproc $®$ R25 (VDW).

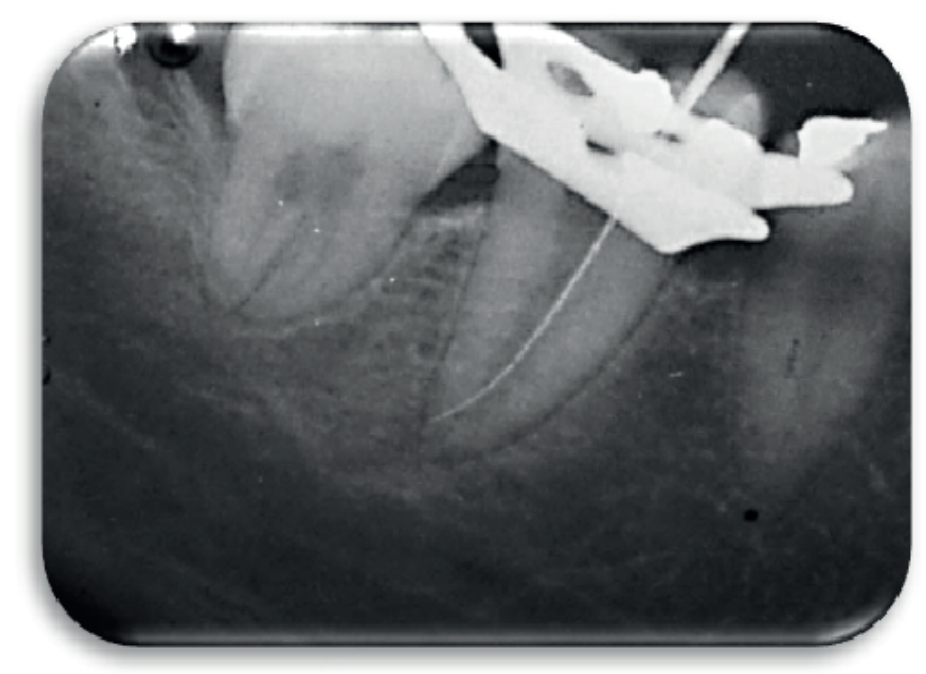

Figura 3. Radiografía de conductometría y verificación con el localizador apical Raypex 6 (VDW)

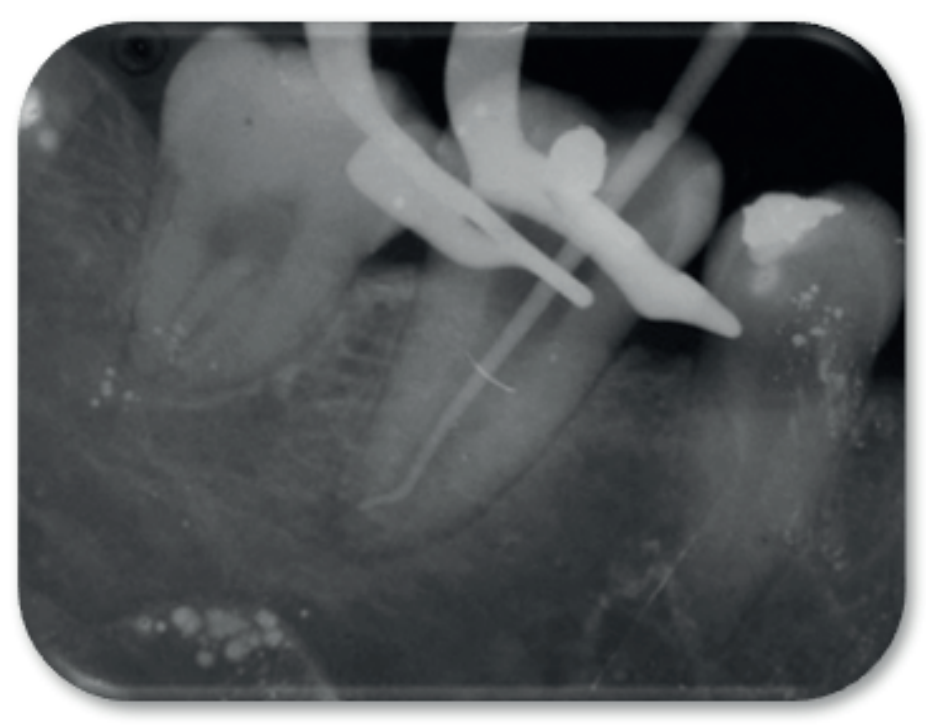

Figura 4. Radiografía de conometría pieza 47 
Se colocó hidróxido de calcio Apexcal® (Ivoclar-Vidadent) como medicación intraconducto y el sellado provisional con cemento de policarboxilato de zinc. Después de 7 días, se seleccionó el cono de gutapercha principal Reciproc® R25 (VDW) y se verificó su adaptación a través de la radiografía de conometría (Figura 4). Se irrigó con $3 \mathrm{ml}$ de EDTA al 17\% durante 5 minutos y fue neutralizado con suero fisiológico, se secó el conducto con puntas de papel Reciproc®(VDW). Posteriormente se desinfectó el cono de gutapercha principal con hipoclorito de sodio al 5,25\% y se obturó con la técnica de compactación de onda continua de calor (Beefil 2 in 1 - VDW) para mejorar la adaptación del material obturador en el sistema de conductos (Figura 5).

Transcurrido 3 años, al control clínico y radiográfico del tratamiento endodóntico y restaurador el paciente se encuentra asintomático clínica y radiográficamente con ausencia de lesión apical. La radiografía panorámica revela variación anatómica en el molar contralateral (Figura 6).

\section{Discusión}

La anatomía del segundo molar mandibular y del primer molar mandibular, por lo general, tienen dos conductos mesiales y un conducto distal, cuando se presenta una variación anatómica con raíces fusionadas, se presume de la existencia de una variación anatómica, conducto único o conducto en forma de $\mathrm{C}^{1,7}$.

Los conductos en forma de C representan el 8,5\% de las variaciones anatómicas más frecuentes en los segundos molares mandibulares, este último se caracteriza por tener un solo orificio con forma acintada que describe un arco de 180 grados o más y que presenta mayor prevalencia en los segundos molares inferiores ${ }^{3,18}$.

Los sistemas de conductos radiculares denominados "en forma de C" deben exhibir las tres características siguientes; raíces fusionadas, surco longitudinal en la superficie de la raíz lingual y/o bucal y al menos una sección transversal del conducto debe tener la configuración $\mathrm{C} 1, \mathrm{C} 2$ o $\mathrm{C} 3{ }^{19}$. El tratamiento descrito presentó

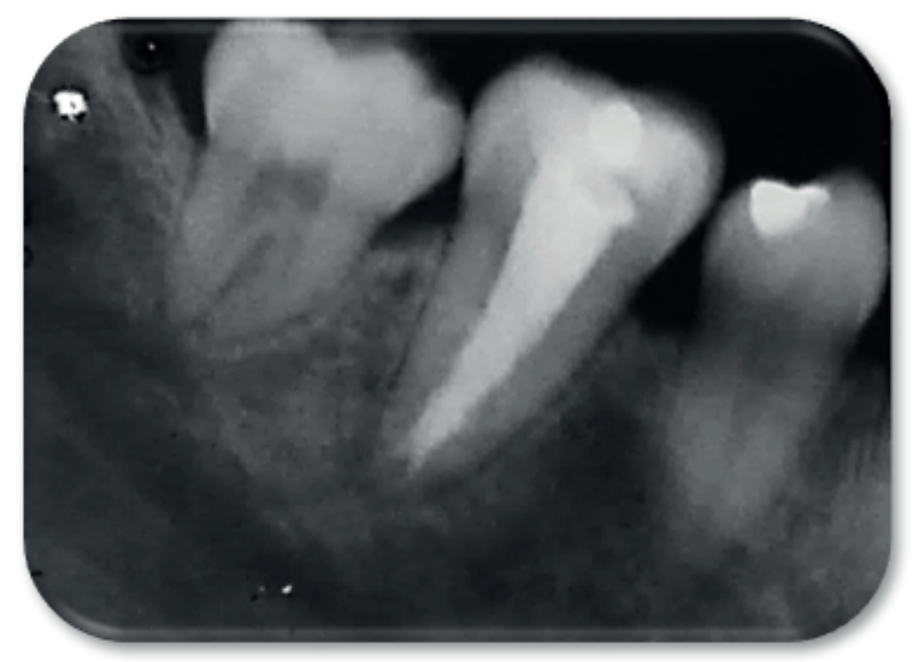

Figura 5. Radiografía de obturación final pieza 47

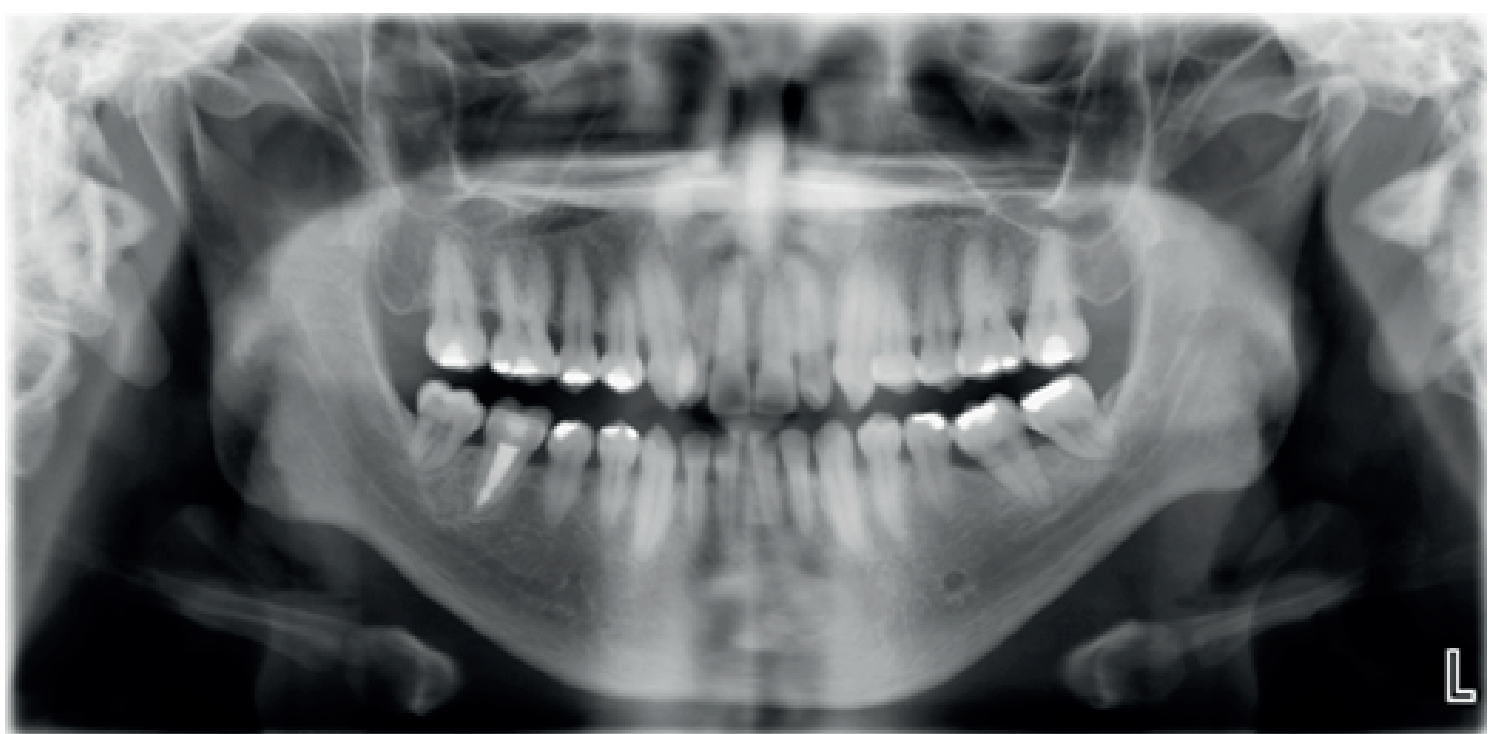

Figura 6. Radiografía panorámica de control a los 3 años 
fusión radicular y a nivel cervical una configuración C tipo I, a nivel de tercio medio presentó una configuración categoría IV y a nivel apical nuevamente describe la forma tipo I, de acuerdo a la evaluación de los cortes axiales de las imágenes obtenidas por la CBCT.

Se asume que la gran diversidad racial que caracteriza a la población peruana es un factor etiológico, pues de acuerdo al estudio realizado por Quijano et al. ${ }^{11}$ en la población peruana, la prevalencia de conductos en forma de $\mathrm{C}$ en segundos molares mandibulares es alta (40,5\%), debido probablemente a la ascendencia china.

Las teorías sobre la morfología en forma $\mathrm{C}$ son diversas y las más conocidas son: la falla de la vaina epitelial de Hertwig o formarse por la coalescencia debido a deposición del cemento con el tiempo ${ }^{4}$. Actualmente, se sabe que el gen que da origen a raíces y conductos en forma de $\mathrm{C}$ es atribuido al cromosoma $5^{7}$.

El uso de la magnificación es de gran ayuda para poder identificar este tipo de casos, debido a que la ubicación de la pieza dentaria y el grado de apertura bucal del paciente dificulta la visibilidad. En el presente caso se utilizó la magnificación $2.5 \mathrm{x}$, lo cual permitió la visualización de la anatomía del piso de la cámara.

Respecto a la evaluación radiográfica de esta variación anatómica Kato et al. ${ }^{7}$ y Wang et al. ${ }^{15}$ describen varios estudios que demuestran que el conducto radicular en forma de $\mathrm{C}$ no se observa fácilmente usando radiografías preoperatorias y en el caso de ser identificadas, este tipo de morfología es bilateralmente simétrica y se recomienda que también debiera ser inspeccionada la pieza del lado opuesto. Siguiendo estas directrices, en el caso reportado se evaluó la presencia de bilateralidad en piezas contralaterales, la cual fue confirmada a través de la radiografía panorámica de control.

Por la dificultad de precisar con certeza la variación anatómica de forma radiográfica, se realizó la CBCT (Planmeca Romexis) de campo pequeño de la pieza dental 47, lo cual ayudó a identificar y precisar el subtipo de variación.

Se utilizó de forma adicional la clasificación propuesta por Vertucci ${ }^{17}$, siendo de tipo V, pues al igual que Sharma ${ }^{20}$, se coincide que es difícil de determinar a qué tipo de clasificación pertenece el conducto en $\mathrm{C}$, por eso pueden ser descritos con la clasificación de Vertucci o de Weine.

La preparación biomecánica es un factor importante para el éxito del tratamiento endodóntico, por lo cual se debe tener en cuenta que los conductos en forma de $\mathrm{C}$ tienen distintos espesores a nivel de las paredes, especialmente de las linguales, que habitualmente son más delgadas que las paredes bucales en los diferentes niveles a lo largo del conducto, haciendo que el tratamiento sea más complejo ${ }^{21}$, por lo que se debe tener cuidado con los instrumentos de mucha conicidad o taper pues el riesgo de perforaciones es alta.

Respecto a la obturación del sistema de conductos radiculares en conductos en forma de $\mathrm{C}$ se recomiendan técnicas de obturación termoplastificada que aseguren el sellado hermético de las irregularidades presentes en este tipo de configuración.

Como consideración final, los endodoncistas debemos estar familiarizados con la apariencia clínica y radiográfica de la raíz de los conductos en forma de $\mathrm{C}$ para poder establecer un diagnóstico correcto, y así poder planificar un adecuado plan de tratamiento haciendo uso de los recursos tecnológicos que acrecientan la probabilidad de éxito a largo plazo.

\section{Referencias bibliográficas}

1. Figún ME, Garino R. Sistema Dentario. En: García P, editores. Anatomía odontológica funcional y aplicada. Buenos Aires: El ateneo; 2003.p. 186-361.

2. Roy A, Velmurugan N, Suresh N. Mandibular second molar with a single root and a single canal: Case series. J Clin Diagnostic Res. 2013;7(11):2637-8. DOI: 10.7860/JCDR/2013/6172.3635

3. Cleghorn BM, Goodacre CJ, Christie W. Morphology of teeth and their root canal systems. En: Ingle JI, Backland LK, Baumgartner CJ, editores. Ingle's Endodontics 6. Hamilton. BC: Decker INC; 2008. p.151 - 220.

4. Elumalai D, Kumar A, Tewari RK, Mishra SK, Andrabi SM, Iftekhar H, Alam S. Management of C-shaped root canal configuration with three different obturation systems. Eur J Gen Dent. 2015;4(2):25-8. DOI: $10.4103 / 2278-9626.149685$

5. Hargreaves KM. Cohen S. Vías de la Pulpa. 10th ed. St. Louis; Elsevier editor; 2011.

6. Kirilova J, Topalova-Pirinska S. C-Shaped Configuration of the Root Canal System - Problems and Solutions. J IMAB. 2014;20(1):504-9. DOI: 10.5272/jimab.2014201.504

7. Kato A, Ziegler A, Higuchi N, Nakata K, Nakamura H, Ohno N. Aetiology, incidence and morphology of the C-shaped root canal system and its impact on clinical endodontics. Int Endod J. 2014;47(11):1012-33. DOI: 10.1111/iej.12256. Epub 2014 Mar 31.

8. Anand S, Christalin R, Rejula F, Anulekh Babu, Sreeja J, Gopinath AS. C-Shaped Canal Systems: a Review. J Int Oral Care Reserch. 2015;3(4):58-66.

9. Thakar SS, Motghare V, Prabhakar I, KK, Gupta B. Gupta N. Bilateral Presence of a Single Root in Mandibular Second Molars having a Single Non-Conical Canal Configuration : A Rare Case Report. 2014;1(3):31-4.

10. Przesmycka A, Tomczyk J. Differentiation of root canal morphology - a review of the literature. Anthropol Rev. 2016;79(3):221-39. DOI: 10.1515/anre-2016-0018

11. Quijano S, García C, Rios K, Ruiz V, Ruíz A. Sistema de conducto radicular en forma de $\mathrm{C}$ en segundas molares mandibulares evaluados por tomografía cone beam. Rev Estomatol Hered. 2016;26(1):28-36. Available from: http://www.upch.edu.pe/vrinve/dugic/revistas/index. php/REH/article/view/2818/2679

12. Min Y, Fan B, Cheung GSP, Gutmann JL, Fan M. C-shaped Canal System in Mandibular Second Molars Part III: The Morphology of the Pulp Chamber 
Floor. J Endod. 2006;32(12):1155-9. DOI: 10.1016/j. joen.2006.07.004

13. Fan W, Fan B, Gutmann JL, Fan M. Identification of a C-shaped canal system in mandibular second molars. Part III. Anatomic features revealed by digital subtraction radiography. J Endod. 2008;34:1187-90. DOI: 10.1016/j.joen.2008.06.013.

14. Bansode P V, Wavdhane MB, Pathak SD, Rana HB. C-Shaped Root Canal Anatomy: A Literature Review. Int J Med Sci Clin Invent. 2017;4(41):2538-43. DOI: 10.18535/ijmsci/ v4i1.03

15. Wang Y, Guo J, Yang HB, Han X, Yu Y. Incidence of C-shaped root canal systems in mandibular second molars in the native Chinese population by analysis of clinical methods. Int J Oral Sci. 2012;4(3):161-5. DOI: 10.1038/ijos.2012.42
16. Fernandes M, de Ataide I, Wagle R. C-shaped root canal configuration: A review of literature. J Conserv Dent 2014;17:312-9.

17. Vertucci F. Root canal anatomy of the human permanent teeth. Oral Surgery Oral Med Oral Pathol. 1984;58:58999. DOI: 10.1016/0030-4220(84)90085-9

18. Nageswar-Rao R. Endodoncia Avanzada. 1ra edición. Amolca, editor. Caracas: Actualidades Medicas; 2011.

19. Chockattu SJ, Deepak BS. Categorization and management of various types of C-shaped canals: Two case reports. J Clin Diagnostic Res. 2017;11(9):6-8. DOI: 10.7860/JCDR/2017/28122.10623

20. Sharma S: Single Rooted Mandibular Second Molars with Single Canal. J Dentofacial Sci. 2013;2(3):27-30.

21. Chokshi S, Mehta J, Chokshi P, Vaidya R. Morphological variations in the root canal system of mandibular second molar: a case series. Endodontology. 2013;25:135-8. 
